\title{
Potentially inappropriate medications and potential prescribing omissions. Identification and relevance
}

\author{
Soraia Azevedo ${ }^{1}$, Rosana Maia², Emília Guerreiro² \\ ${ }^{1}$ ULSAM - Ponte de Lima. ${ }^{2}$ ULSAM - Viana do Castelo
}

\begin{abstract}
Introduction: Europe presents $19 \%$ of the population aged 65 or over (elderly), who are especially susceptible to inadequate prescriptions (potentially inappropriate medications (PIM) and potential prescribing omissions (PPO)), identified by the STOPP and START criteria. This study has as main objectives the identification of PIM and PPO and associated factors. Material and Methods: This is a cross sectional study, using a sample of 254 elderly individuals from the Internal Medicine Service. Results: $81.9 \%$ of patients are polymedicated, $72.4 \%$ have at least one PIM and $57.5 \%$ have at least one PPO. The most frequently identified PIM was benzodiazepine and PPO was the anti-pneumococcal vaccine. There was a direct correlation between the number of PIM and chronic medication $(r(254)=0.348, p<0.001)$ and inverse with the Katz scale (dependence) $(r(254)=-0.324, p<0.001)$. In the cases of the domicile it was verified association between PIM and the days of internment $(U=3653, p=0.025)$. PIM were associated with death in less than 6 months after discharge $(U=3396, p=0.007)$ and the presence of intercurrences at admission $(U=5766, p=0.005)$. There is a relationship between the number of co-morbidities and having at least one PIM $(U=5378, p=0.041)$ or at least one PPO ( $U=6271, p=0.005)$. Diabetes mellitus (DM) type 2, neurological and psychiatric disease are associated with PIM, while obesity, DM type 2, arterial hypertension, dyslipidemia and cardiac pathology with PPO. Discussion and Conclusion: In a population that is older each year, with more comorbidities and more polymedicated, PIM and PPO are increasingly relevant.

Keywords: START and STOPP criteria; polymedication; potentially inappropriate medications (PIM); potential prescribing omissions (PPO).
\end{abstract}

\section{INTRODUCTION}

In 2017, Europe had about 19\% of the population with 65 years old or over (elderly) 1 . With age comorbidities increase and physiological changes occur that modify the pharmacokinetics and pharmacodynamics of the drugs ${ }^{2}$. Therefore, this group is especially susceptible to polymedication, drug interactions and adverse effects, thus have an increased risk of inadequate prescriptions. ${ }^{3}$ Inappropriate prescriptions include potentially inappropriate medications (PIM) or omissions (PPO) $)^{4,5}$. In order to be possible to identify PIM, STOPP (Screening Tool of Older PersonsÂ' Prescriptions) were created, while PPO are identified by the START criteria (Screening Tool to Alert to Right Treatment). The STOPP / START criteria were primarily published in 2008 and updated in 2015, validated by a consensus of European experts ${ }^{5}$. Since PIM and PPO are associated with several adverse effects, it is important to determine the prevalence, identify the contributors, and review prescribing indications, so that it is possible the optimization of the therapy ${ }^{6,7,8}$. Another possible consequence of aging is dependence, which is associated with an increase of costs and decrease of life quality. The Katz scale allows the evaluation of the elderly's autonomy to perform the basic and crucial activities of daily life, called Basic Activities of Daily Life (BADL) ${ }^{9}$.

The prevalence of PIM and PPO is high, varying according to the place where the evaluation is performed. In primary care PIM values ranged from $21.4 \%$ to $42.0 \%$, and PPO from $22.7 \%$ to $32.0 \%^{10,11}$. In secondary health care PIM approaches $34.5 \%$ and PPO $57.9 \%^{12}$. In the institutionalized elderly, the prevalence of PIM can exceed $75.0 \% \cdot{ }^{13}$ n Portugal and others European countries studies on this subject are scarce, with small samples and focus mostly on the population of nursing homes ${ }^{14}$. Thereby the need for new more embracing studies.

At the level of PIM associations, there are few factors studied, these include the absence of dementia, severe comorbidities and multiple medications ${ }^{13}$.

So it is fundamental to approach this theme in our community, increasingly aged. This study has as main objectives the identification of PIM and PPO and associated factors.

\section{MATERIAL AND METHODS}

This is a cross sectional observational study. The research protocol was approved by the Ethics Committee of the Hospital: Unidade Local de Saúde do Alto Minho (ULSAM).

Patients with 65 or more years of age hospitalized on the Medical Service of ULSAM from January 01 to November 30,2017 , randomly selected were included. Patients in palliative care and deaths during hospitalization were excluded because it was not possible to evaluate the PPO introduced and PIM suspended. In bedridden and end-of-life, PPO was not considered, except for symptomatic treatments, as the remaining therapeutic incarceration was considered.

There is no consensus about the definition of polymedication, but the value of 5 or more drugs is the most consensual, and this was the value attributed ${ }^{15}$. 
The degree of dependence was assessed by the Katz scale before the acute phase, on admission and on discharge ${ }^{9}$.

To evaluate PIM and PPO, the STOPP/START criteria were used, but updated according to the latest recommendations. Particularly in regard to hypocoagulation in atrial fibrillation ${ }^{16}$, to the prescription of proton pump inhibitors (PPIS) ${ }^{17}$ and to anti-pneumococcal vaccination ${ }^{18}$.

The classification of the professions was made by the Portuguese classification of professions 2010. The evaluation of the number of external consultations only took into account the consultations made by doctors.

It was considered excessive alcohol consumption, if superior to the recommended one (up to $10 \mathrm{gr}, 20 \mathrm{gr}$ if man from 18 to 64 years); addiction was considered as a psychiatric disease $^{19}$.

\section{STATISTICAL ANALYSIS}

Statistical analysis was performed in the Statistical Package for the Social Sciences (SPSS) version 24. The descriptive statistical analysis was performed by the evaluation of the absolute and relative frequencies of the categorical variables and by the calculation of the mean, median and standard deviation in the continuous variables. The study of the normality of the distribution of continuous variables was made with the Kolmogorov-Smirnov Test (KS) and Shapiro-Wilk test (SW). In the comparison of means between groups, for the variables with normal distribution, the Student $t$ Test (t) was applied. In the ones that a normal distribution was not verified and for the ordinal independent variables with more than 3 cat- egories, The Mann-Whitney U test (U) was applied, but only if the independent variable was binary. If it had more than 2 categories, The Kruskal-wallis H Test was used. In case it was dependent variables, the Wilcoxon Test was used. For the evaluation of the relationship between two categorical variables, the Qui-Quadrado Test ( $\mathrm{x} 2)$ was used, and when its assumptions were not assured Fisher's Exact Test was used. To measure the degree of correlation between two quantitative variables Pearson's Correlation Coefficient was used. The value of statistical significance for all tests was defined as 2 -sided $p<0.050$.

Results:The age of the 254 cases ranged from 65 to 99 years, with a median of 80 years ( $S D=7.97)$, not presenting a normal distribution, $55.5 \%$ of the cases are female and $44.5 \%$ are male. $78.7 \%(200)$ of the cases come from home/with families, $15.4 \%$ (39) of households, $3.15 \%$ (8) from host families and $2.75 \%$ (7) from national integrated network of integrated care (NINIC). The sample was characterized by the degree of dependence (by the Katz scale) before the acute phase, at admission, at discharge and its variation (Table 1). Patients were more dependent after hospitalization than before the acute phase $(Z=-5.57, p<0.001)$, but were less dependent at discharge than at admisson.

The number of comorbidities ranged from 1 to 16 , with a median of 6.5. The comorbidities were characterized (Table 2).

The number of drugs prescribed ranged from 0 to 19, with a median of 8 , out of a total of 1953 medications usually taken by 254 patients. $81.9 \%$ (208 cases) were polymedicated and only $3(1.18 \%)$ of the patients had no chronic medica-

Table 1. Characterization of the sample regarding the degree of dependence.

\begin{tabular}{|l|c|c|c|}
\hline Katz Scale & $\begin{array}{c}\text { Before acute } \\
\text { phase of illness }\end{array}$ & Admission & Discharge \\
\hline Total dependence (Katz: 0) & $63(24.8 \%)$ & $87(34.3 \%)$ & $77(30.3 \%)$ \\
\hline Severe dependence (Katz: 1) & $12(4.72 \%)$ & $9(3.54 \%)$ & $13(5.12 \%)$ \\
\hline Severe dependence (Katz: 2) & $20(7.87 \%)$ & $19(7.48 \%)$ & $19(7.48 \%)$ \\
\hline Moderate dependence (Katz: 3) & $14(5.51 \%)$ & $14(5.51 \%)$ & $11(23.4 \%)$ \\
\hline Moderate dependence (Katz: 4) & $25(9.84 \%)$ & $43(16.9 \%)$ & $34(13.4 \%)$ \\
\hline Slight dependence (Katz: 5) & $23(9.06 \%)$ & $26(10.2 \%)$ & $23(9.05 \%)$ \\
\hline Independence (Katz: 6) & $97(38.2 \%)$ & $56(22.0 \%)$ & $77(30.3 \%)$ \\
\hline Variation of dependence & & & \\
\hline Katz Scale at discharge $<$ Katz Scale before acute phase of illness & & $40(15.7 \%)$ & \\
\hline Katz Scale at discharge $>$ Katz Scale before acute phase of illness & & $214(84.3 \%)$ \\
\hline Katz Scale at discharge $=$ Katz Scale before acute phase of illness & & \\
\hline
\end{tabular}


Table 2. Characterization of the sample regarding comorbidities.

\begin{tabular}{|c|c|}
\hline Identification of the comorbidities & (n (\%valid)) \\
\hline Obesity or overweight BMl $>25 \mathrm{~kg} / \mathrm{m}^{2}$ & $68(26.8 \%)$ \\
\hline Type 2 diabetes Mellitus & $97(38.2 \%)$ \\
\hline Arterial hypertension & $195(76.8 \%)$ \\
\hline Dyslipidemia & $143(56.3 \%)$ \\
\hline Hyperuricemia & $34(13.4 \%)$ \\
\hline Excessive consumption of alcohol & $20(7.87 \%)$ \\
\hline $\begin{array}{l}\text { Smoking } \\
\text { Non smoker } \\
\text { Former smoker } \\
\text { Smoker }\end{array}$ & $\begin{array}{l}199(82.2 \%) \\
36(14.9 \%) \\
7(2.90 \%)\end{array}$ \\
\hline $\begin{array}{l}\text { Pulmonary Pathology * } \\
\text { COPD clinical / probable diagnosis } \\
\text { COPD established } \\
\text { Others }\end{array}$ & $\begin{array}{l}78(30.7 \%) \\
27(34.6 \%) \\
24(30.8 \%) \\
27(34.6 \%)\end{array}$ \\
\hline $\begin{array}{l}\text { Heart Pathology } \\
\text { Atrial fibrillation } \\
\text { Cardiac insufficiency } \\
\text { Valvular Disease } \\
\text { Ischemic Heart Disease } \\
\text { Pacemaker } \\
\text { Prosthesis } \\
\text { Others }\end{array}$ & $\begin{array}{l}146(57.5 \%) \\
90(61.6 \%) \\
76(52.1 \%) \\
37(25.3 \%) \\
28(19.2 \%) \\
16(11.0 \%) \\
11(6.59 \%) \\
19(15.39 \%)\end{array}$ \\
\hline $\begin{array}{l}\text { Neurological Pathology } \\
\text { Dementia, including Alzheimer's disease } \\
\text { Ischemic stroke } \\
\text { Hemorrhagic stroke } \\
\text { Parkinson's disease or parkinsonism } \\
\text { Others }\end{array}$ & $\begin{array}{l}119(46.9 \%) \\
61(51.3 \%) \\
50(42.0 \%) \\
10(8.40 \%) \\
14(11.8 \%) \\
25(21.00 \%)\end{array}$ \\
\hline $\begin{array}{l}\text { Psychiatric Pathology } \\
\text { Depression } \\
\text { Alcohol addiction } \\
\text { Other psychiatric conditions }\end{array}$ & $\begin{array}{l}71(28.0 \%) \\
57(80.3 \%) \\
8(11.3 \%) \\
7(9.86 \%)\end{array}$ \\
\hline $\begin{array}{l}\text { Hematologic Pathology } \\
\text { Anemia } \\
\text { Other hematological diseases }\end{array}$ & $\begin{array}{l}51(20.1 \%) \\
37(72.5 \%) \\
18(35.32 \%)\end{array}$ \\
\hline Malignant neoplasm & $56(22.0 \%)$ \\
\hline Dizziness & $12(4.72 \%)$ \\
\hline Benign Prostatic Hyperplasia & $37(32.7 \%)^{\star \star}$ \\
\hline Chronic Kidney Disease & $50(19.7 \%)$ \\
\hline Chronic Hepatic Disease & $6(2.36 \%)$ \\
\hline Other comorbidities & $175(68.9 \%)$ \\
\hline
\end{tabular}

* Excluded pulmonary neoplasms included in neoplastic diseases

** Percentage of the number of men

PIM: Potentially Inappropriate Medications; PPO: Potential Prescribing Omissions; BMI: body mass index; COPD: chronic obstructive pulmonary disease; OSA: obstructive sleep apnea; TAVI: transcatheter aortic valve implantation. tions. The PIM number ranged from 0 to 5 , with a median of 1 , in a total of 326 , and $72.4 \%$ (184) of the patients have at least one PIM prescribed. The PPO number ranged from 0 to 4 , with a median of 1 , in a total of 203 , and $57.5 \%$ (146) of the patients have at least one PPO. PIM and PPO were identified (Table 3). Excluding vaccinations of PPO, however, $28 \%$ of patients (71) had at least one PPO.

There was no correlation between age and PIM number $(r(254)=0.019, p=0.760)$ nor PPO $(r(254)=0.80, p=0.203)$, however excluding vaccinations of PPO was a positive correlation between age and PPO $(r(254)=0.131, p=0.037)$. There is a correlation between greater dependence (smaller Katz scale) and more PIM ( $r(254)=-0.324, p<0.00)$ and, excluding vaccinations, PPOs were inversely associated with dependence $(r$ $(254)=-0.191, p=0.002)$. There were no statistically significant differences between the previous profession and the PIM number (Fisher exact test $=12.55, p=0.115$ ), or PPO (Fisher exact test $=10.23, p=0,258)$. Also schooling has no association with PIM (Fisher exact test=4.90, $p=0.717$ ) nor with PPO (Fisher exact test=8.75, $p=0.207$ ).

Of the hospitalized patients with over 65 years, $17.7 \%$ had prostration as the cause of admission, $76.0 \%$ were patients taking benzodiazepines, antipsychotics and or tricyclic antidepressants, even so, of which $26.3 \%$ had even 2 or more classes of drugs with sedative effect.

Of the patients, $31.7 \%$ have a diagnosis of presumably bacterial pneumonia or acute chronic obstructive pulmonary disease (COPD). Of these, 35.6\% had indication to make an anti-pneumococcal vaccine.

The days of hospitalization ranged from 1 to 46 days, with a median of 8 days. The number of days of hospitalization was not correlated with the number of PIM $(r(254)=0.062$, $p=0.323)$ neither PPO $(r(254)=0.096, p=0.128)$. However, when only the patients from home were evaluated, there were differences in the days of hospitalization $(\mathrm{U}=3653$, $p=0.025)$. That is, the PIM led to longer hospitalizations.

There was re-hospitalization in $5.12 \%$ of the cases (less than 5 days after discharge), 10,6\% were hospitalized between 5 and 30 days after discharge and $7.1 \%$ between 1 and 6 months after discharge. However, the number of days for new hospitalization was not correlated with the number of PIM ( $r(254)=-0.041, p=0.518)$ or PPO $(r(254)=0.094$, $\mathrm{p}=0.136)$.

There was no relationship between the number of PIM and hospitalizations in the last 12 months $(U=7002, p=0.482)$ or PPO ( $=6517, p=-0.097)$.

Up to 6 months after a high frequency of 43 deaths (16.9\% of the 254 cases), $20.7 \%$ of the total cases had less than one PIM and $7.17 \%$ of those who had no PIM. There is a relation between PIM number and death in less than 6 months after discharge $(U=3396, p=0.007)$, but this was not verified with PPO $(U=4496, p=-0.921)$. 
Table 3. Identification of PIM and PPO.

\begin{tabular}{|c|c|c|c|}
\hline \multicolumn{3}{|l|}{ PIM and justification } & (n (\%valid)) \\
\hline \multicolumn{3}{|l|}{ Benzodiazepines more than 4 weeks } & $79(31.1 \%)$ \\
\hline \multicolumn{3}{|c|}{ PPI without gastric disease, no history of complicated ulcer, without more than 2 risk factors or for more than 8 weeks } & $77(30.3 \%)$ \\
\hline \multicolumn{3}{|c|}{ Other antipsychotics than quetiapine or clozapine } & $23(9.06 \%)$ \\
\hline \multicolumn{3}{|c|}{ Antiaggregation as primary prevention (without known arterial disease) (1 of the cases with associated anaemia) } & $22(8.66 \%)$ \\
\hline \multicolumn{3}{|c|}{ Association between antiaggregant and hypocoagulant or double antiaggregation without clinical criteria } & $14(5.51 \%)$ \\
\hline \multicolumn{3}{|c|}{ Risperidone, in addition to having extrapyramidal effects, is associated with an increased risk of stroke } & $12(4.72 \%)$ \\
\hline \multicolumn{3}{|c|}{ Antidepressants in patients without depressive symptoms } & $11(4.33 \%)$ \\
\hline \multicolumn{3}{|c|}{ Thiazide or thiazide-like diuretic in patients with history of gout } & $11(4.33 \%)$ \\
\hline \multirow{4}{*}{$\begin{array}{l}\text { Drugs unsuitable for renal function } \\
\text { (GFR: } \mathrm{ml} / \mathrm{min} / 1.73 \mathrm{~m} 2 \text { ) }\end{array}$} & Dapagliflozin with GFR $<60$ & 1 & \multirow{4}{*}{$3.15 \%$} \\
\hline & NSAIDs with GFR $<50$ & 2 & \\
\hline & Metformin with GFR $<30$ & 3 & \\
\hline & Allopurinol 300mg with advanced DRC & 2 & \\
\hline \multicolumn{3}{|c|}{ Prevention of Cerebrovascular or Cardiovascular Disease in bedridden and life expectation less than a year } & $7(2.76 \%)$ \\
\hline \multicolumn{3}{|c|}{ Sulfunyloreas of long duration of action, high risk of hypoglycemia } & $7(2.76 \%)$ \\
\hline \multicolumn{3}{|c|}{ Other antidepressants other than SRls or new mechanisms of action (safer and less pharmacological interactions) } & $6(2.36 \%)$ \\
\hline \multicolumn{3}{|l|}{ Sedative drugs without clinical criteria } & $5(1.97 \%)$ \\
\hline \multicolumn{3}{|c|}{ Atrial fibrillation with rhythm control without frequency control } & $5(1.97 \%)$ \\
\hline \multicolumn{3}{|c|}{ Antianginal agents without known angina or ischemic heart disease } & $5(1.97 \%)$ \\
\hline \multicolumn{3}{|c|}{ Systemic corticosteroid in COPD without optimization of inhaled therapy } & $4(1.57 \%)$ \\
\hline \multicolumn{3}{|c|}{ Fenofibrate / ezetimibe without statin (statins are the first line in the treatment of dyslipidemia) } & $4(1.57 \%)$ \\
\hline \multicolumn{3}{|c|}{ Drugs that aggravate parkinsonism and $\mathrm{Cl}$ association with $\mathrm{MAOI}$ (eg: cinnarizine, mirtazapine) } & $4(1.57 \%)$ \\
\hline \multicolumn{3}{|c|}{ Strong opioids without further attempts to control pain } & $3(1.18 \%)$ \\
\hline \multicolumn{3}{|c|}{ Central-acting antihypertensives (others safer classes are available) } & $3(1.18 \%)$ \\
\hline \multicolumn{3}{|l|}{ Others } & $16(6.27 \%)$ \\
\hline \multicolumn{3}{|l|}{ PPO and justification } & (n (\%valid)) \\
\hline \multicolumn{3}{|l|}{ Anti-pneumococcal vaccine * } & $93(36.6 \%)$ \\
\hline \multicolumn{3}{|c|}{ Vitamin D in institutionalized patients with a high risk of falls } & $27(10.6 \%)$ \\
\hline \multirow{2}{*}{ Anti-resorptive therapy } & For previous frailty fracture & 17 & \multirow{2}{*}{$7.87 \%$} \\
\hline & By known osteoporosis & 3 & \\
\hline \multicolumn{3}{|l|}{ Flu's vaccine } & $19(7.48 \%)$ \\
\hline Inhaled bronchodilators or corticost & ts with COPD & & $12(5.51 \%)$ \\
\hline Hypoagulation in patients with atria & nout contraindications & & $7(2.76 \%)$ \\
\hline Laxatives in patients receiving opioi & sstipation & & $6(2.36 \%)$ \\
\hline Statin by previous ischemic stroke & idemia & & $4(1.57 \%)$ \\
\hline Others & & & $15(7.06 \%)$ \\
\hline
\end{tabular}

PIM: Potentially Inappropriate Medications; PPO: Potential Prescribing Omissions; PPI: Proton Pump Inhibitors; GFR: glomerular filtration rate; NSAIDs: non steroid antiinflammatory drugs; COPD: chronic obstructive pulmonary disease; 
Patients with the highest number of PIM had plus intercurrences during the hospitalization period $(U=5766, p=0.005)$, the cases that had at least one PIM $38.0 \%$ had intercurrences during hospitalization, while the number of PPO did not influenced the occurrence of intercurrences $(U=7024$, $\mathrm{p}=0.641$ ).

A total of 326 PIM were obtained, with 182 (55.8\%) being suspended and total of 203 PPO, 156 (76.8\%) were introduced. But neither the PIM suspension ( $U=3305, p=0.303)$ nor the PPO introduction $(U=1693, p=0.544)$ significantly affected the time to re-hospitalization after discharge.

There is a significant relation between the number of comorbidities and the minimum of one PIM $(U=5378, p=0.041)$ and the minimum of one PP0 $(U=6271, p=0.005)$. The patients with more comorbidities had a greater probability of PIM and PPO. A Diabetes Mellitus (DM) type 2, a neurological and psychiatric disorder, is associated with the number of PIM and obesity, DM type 2, arterial hypertension, dyslipidaemia and cardiac pathology with PPO numbers (Table 4).

A correlation between the number of external consultations and the number of PIM ( $r(254)=-0.067, p=0.290)$ or PPO $(r(254)=-0.100, p=0.113)$ was not verified. There was a direct correlation between the number of drugs usually taken by patients and the number of PIM $(r(254)=0.348$, $p<0.001)$, but not with PPO $(r(254)=0.088, p=0.163)$.

\section{DISCUSSION}

The age and gender distribution of this sample is similar of the European population over 65 years of age, in which $57.9 \%$ are women and $42.1 \%$ are men ${ }^{1}$. In the last years we have witnessed an increase in the average life expectancy, being in 2015, 83.9 years for women and 77.7 years for men ${ }^{20}$. But it is not enough to continue giving years to life without giving life to years. In our sample, nearly one-quarter of the patients were already fully dependent and $37.0 \%$ were partially dependent before admission, higher values of dependence than those reported for developed societies, such as our country, and with significant worsening after discharge ${ }^{21}$. In this study there was a direct relation between the degree of dependence and the number of PIM. The question is whether these are inadequate because patients no longer need them or if they have contributed in any way to the degree of dependence. Comorbidities are also common, with a median of 6,5 per patient and are related both to PIM and to PPO. That is, people with more comorbidities have more PIM and PPO. Since DM type 2, neurological and psychiatric diseases are associated with PIM. Other studies show an association with absence of dementia, severe comorbidities and multiple medications ${ }^{13}$.

There are no published studies that show the comorbidities associated with PPO. This study demonstrates the association with obesity, DM type 2, arterial hypertension, dyslipidaemia and cardiac pathology. That is of special emphasis since this pathologies are very common among the elderly population.

Table 4. Association between comorbidities and PIM and PPO.

\begin{tabular}{|l|c|c|}
\hline & PIM Number & $P P 0$ Number \\
\hline Obesity / Overweight & $U=6254, p=0.889$ & $U=4850, p=0.002$ \\
\hline Arterial hypertension & $U=5670, p=0.862$ & $U=3408, p<0.001$ \\
\hline Diabetes Mellitus type & $U=6427, p=0.036$ & $U=6237, p=0.011$ \\
\hline Dyslipidemia & $U=7731, p=0.713$ & $U=6691, p=0.021$ \\
\hline Excessive consumption of alcohol & $U=1951, p=0.278$ & $U=2012, p=0.365$ \\
\hline Smoking (non-smoker, ex-smoker, smoker) & $\mathrm{H}(2)=2.78, p=0.250$ & $\mathrm{H}(2)=0,149, p=0.928$ \\
\hline Pulmonary disease & $U=6505, p=0.615$ & $U=6670, p=0.851$ \\
\hline Cardiac disease & $U=7535, p=0.531$ & $U=6455, p=0.008$ \\
\hline Hematologic disease & $U=5007, p=0.708$ & $U=4986, p=0.662$ \\
\hline Neurological disease & $U=7,88, p=0.005$ & $U=7339, p=0.200$ \\
\hline Psychiatric disease & $U=4906, p=0.002$ & $U=6187, p=0.525$ \\
\hline Cancer & $U=4858, p=0.141$ & $U=5533, p=0.981$ \\
\hline Chronic kidney disease & $U=4491, p=0.174$ & $U=4525, p=0.183$ \\
\hline Chronic hepatic disease & $U=624, p=0.484$ & $U=526, p=0.186$ \\
\hline
\end{tabular}

PIM: Potentially Inappropriate Medications; PPO: Potential Prescribing Omissions. 
After being proved in several studies the association of PIM with adverse pharmacological effects, hospitalizations, morbidity and mortality, functional disability and many problems that could be prevented such as falls and confusion and, consequently, associated with more costs $7,8,9,22,23$. There is, moreover, a study that states that about $30 \%$ of hospital admissions are secondary to adverse drug effects ${ }^{22}$. But not only PIM have harmful consequences for health status, PPO may also have. This study uses the STOPP and START criteria so that PPO can be evaluated in addition to PIM. It reinforces the significance of PIM due to its association with the days of hospitalization (in the patients coming from the home), the death in less than 6 months after the discharge and the presence of intercurrences in the hospitalization.

The risks of polymedication and pharmacological interactions are several and known ${ }^{10,24,25}$. In this study $81.9 \%$ of patients are polymedicated, values much higher than those reported in other studies $(20-75 \%)^{25,26}$. Existing a direct correlation between the number of drugs usually taken and PIM. $72.4 \%$ of the cases have at least one PIM and $57.5 \%$ at least one PPO. Values similar to other results of small Portuguese studies about PIM (74.0\%), but not about PPO (29.0\%), which may be due to the clinical guideline standard about pneumococcal vaccine ${ }^{18,27}$. PIM values are much higher than other international studies ${ }^{12,28}$. The most frequently identified PIM were benzodiazepines, PPIs and antipsychotics.

Benzodiazepines are associated with physical dependence, anterograde amnesia and risk of falls. On the other hand, they are also associated with more health spending, even when other health/disease factors are taken into account 29,30 . Antipsychotics (other than quetiapine and clozapine) are associated with a higher risk of stroke, extrapyramidal effects and mortalityy ${ }^{29,32}$. Chronic use of PPIs is associated with an increased risk of pneumonia, enteric infections especially per Clostridium difficile and spontaneous bacterial peritonitis due to acid suppression ${ }^{33,34,35}$.

The most common PPOs were anti-pneumococcal vaccine, anti-resorptive therapy and vitamin D. According to the clinical guideline standard vaccination against Streptococcus pneumoniae infections is recommended in immunocompetent patients with chronic heart, liver, renal or respiratory disease, pre-transplantation of organ or donation of bone marrow, DM and in case of cerebrospinal fluid fistulas or cochlear implants. In immunocompromised patients it is recommended for splenic dysfunction, primary immunodeficiency, human immunodeficiency virus (HIV) infection, transplant recipients, active neoplastic disease, and iatrogenic immunosuppression ${ }^{18}$. Anti-reabsorption therapy is recommended in patients with bone densitometry revealing osteoporosis and patients with fragility fracture regardless of the value of the densitometry ${ }^{36}$. Vitamin $D$ should be routinely given to institutionalized elderly people because they have low consumption, low sun exposure and low cutaneous synthesis ${ }^{37}$.
Of hospitalized patients, 31.7\% had a diagnosis of presumably bacterial pneumonia or acute COPD. Of these, $35.6 \%$ were indicated according to the Portuguese standard to have had the anti-pneumococcal vaccine. It should be noted that bedridden and low life expectance patients were taken into account in the percentage of pneumonia diagnosed, but were not considered to be indicated for vaccination regardless of the remaining background. Although vaccination did not have an absolute efficacy it could eventually have made some difference. The cause of admission was prostration in $17.7 \%$ of the cases, it is clinically evident that a significant portion of this prostration may be related to changes in systemic inflammatory response syndrome. Nevertheless, $76.0 \%$ of these patients were taking benzodiazepines, antipsychotics and or tricyclic antidepressants. It is important to emphasize that of these, $26.3 \%$ had even 2 or more classes of drugs with sedative effect.

Having a relation between the number of PIM and the number of medications usually taken, it is worrying the increase in the number of chronic medication in the elderly population either in outpatient, nursing and inpatient patients ${ }^{28}$.

Of a total of 326 PIM, 182 (55.8\%) were suspended and from a total of 203 PPO 156 (76.8\%) were introduced. This, along with the correlation between PIM and the number of medications usually taken, once again shows the need to consider the introduction of new medication. This article comes to prove the relevance of identifying the PPO and introducing them and the PIM and suspending them. But not everything depends on the prescribers, there are PPO patients only because they refuse to do them. Let's look at the example of the flu vaccine that is given for free to all the elderly in Portugal and $7.48 \%$ of the elderly did not do it.

\section{CONCLUSION}

This study, along with others already published, proves the importance of PIM and PPO. In Europe, we have older people who are more dependent, with more comorbidities, more polymedicated. In Portugal we have more PIM than other countries. With aging, improved health care and basic hygiene conditions, we will increasingly have older patients with more comorbidities and therefore with a higher risk of PIM and PPO, which makes this subject even more relevant. We are probably at the point where it is no longer possible to continue to medicate without an international/national strategy for polymedication.

\section{BIBLIOGRAPHY}

1. The share of elderly people continues to increase. Eurostat. Data extracted in May 2018

2. Benson JM. Antimicrobial Pharmacokinetics and Pharmacodynamics in Older Adults. Infect Dis Clin North Am. 2017 Dec;31(4):609-617

3. Scott I, Jayathissa S. Quality of drug prescribing in older patients: is there a problem and can we improve it? Intern Med J. 2010; 40:7-18.

4. Onder $\mathrm{G}$ et al. Strategies to reduce the risk of iatrogenic illness in complex older adults. Age Ageing. 2013 May; 42(3):284-91.

5. OÂ'Mahony $D$ et al. STOPP/START criteria for potentially inappropriate prescribing in older people: version 2.Age Ageing.2015 Mar; 44(2):213-8. 
6. Hamilton H, Gallagher P, Ryan C, Byrne S, OÂ’Mahony D. Potentially inappropriate medications defined by STOPP criteria and the risk of adverse drug events in older hospitalized patients. Arch Intern Med. 2011;171:1013-9

7. OÂ'Connor MN et al. Prevention of Hospital-Acquired Adverse Drug Reactions in Older People Using Screening Tool of Older PersonsÂ' Prescriptions and Screening Tool to Alert to Right Treatment Criteria: A Cluster Randomized Controlled Trial. J Am Geriatr Soc.2016 Aug:64(8):1558-66.

8. Gallagher $\mathrm{PF}, \mathrm{OA} \hat{A}^{\prime}$ Connor MN, $\mathrm{OA} \hat{A}^{\prime}$ Mahony $\mathrm{D}$. Prevention of potentially inappropriate prescribing for elderly patients: a randomized controlled trial using STOPP/START criteria.Clin Pharmacol Ther. 2011;89:845-54.

9. Núcleo de Estudos de Geriatria da Sociedade Portuguesa de Medicina Interna. Avaliação Geriátrica Global "A pedra angular dos Cuidados ao Idoso". 6.

10. Ryan C Potentially inappropriate prescribing in an Irish elderly population in primary care.Br J Clin Pharmacol. 2009 Dec; 68(6): 936-947.

11. Lesende M. et al. Potentiality of STOPP/START criteria used in primary care to effectively change inappropriate prescribing in elderly patients. European Geriatric Medicine, 2013 Nov; Volume 4, Issue 5, 293-298

12. Gallagher PF, OÂ'Mahony D. Screening Tool of Older Persons potentially Inappropriate Prescriptions (STOPP): application to acutely ill elderly patients and comparison with Beer' criteria. Age Ageing.2008; 37:673-9.

13. Tamura BK,Bell CL,Inaba M,Masaki KH. Factors associated with polypharmacy in nursing home residents. Clin Geriatr Med.2012 May;28(2):199-216.

14. da Costa FA et al. Potentially inappropriate medications in a sample of Portuguese nursing home residents: Does the choice of screening tools matter? Int J Clin Pharm.2016 0ct;38(5):1103-11.

15. Hovstadius B, Petersson G. Factors leading to excessive polypharmacy. Clinics in geriatric medicine. 2012;28(2):159-72

16. Kirchhof P. et al. 2016 ESC Guidelines for the management of atrial fibrillation developed in collaboration with EACTS. European Heart Journal, Volume 37, Issue 38, 2016, 2893-2962

17. Lanza FL, Chan FK, Quigley EM Guidelines for prevention of NSAID-related ulcer complications. Am J Gastroenterol. 2009 Mar;104(3):728-38

18. DGS. Vacinação contra infeções por Streptococcus pneumoniae de grupos com risco acrescido para doença invasiva pneumocócica (DIP). Adultos ( $\geq 18$ anos de idade). Norma n011/2015 de 23/06/2015 atualizada a 06/11/2015

19. DGS. Deteção Precoce e Intervenção Breve no Consumo Excessivo de Álcool Norma no030/2012 de 28/12/2012 atualizada a 18/12/2014

20. GBD 2016 Causes of Death Collaborators, Global, regional, and national age-sex specific mortality for 264 causes of death, 1980-2016: a systematic analysis for the Global Burden of Disease Study 2016 Volume 390, No. 10100, 1151-1210, 16 September 2017

21. Heikkien E.: What are the main risk factors for disability in old age and how can disability be prevented? WHO Regional Office for Europe's Health Evidence Network (HEN), 2003.
22. Berdot S, Bertrand M, Dartigues JF, et al. Inappropriate medication use and risk of falls - A prospective study in a large community-dwelling elderly cohort. BMC Geriatr. 2009;9:30.

23. Redston MR et al. Prevalence of Potentially Inappropriate Medication Use in Older Inpatients with and without Cognitive Impairment: A Systematic Review. J Alzheimers Dis. 2017 Dec 16.

24. Maher RL, Hanlon J, and Hajjar ER: Clinical consequences of polypharmacy in elderly. Expert Opin Drug Saf 2014; 13: 57-65

25. Robert L. et al. Clinical Consequences of Polypharmacy in Elderly. Expert Opin Drug Saf. 2014 Jan; 13(1):

26. Onder $\mathrm{G}$ et al.: Polypharmacy in nursing home in Europe: results from the SHELTER study. J Gerontol A Biol Sci Med Sci 2012; 67:698-704.

27. M.M. Moraes, A. Matias, M.A. Soares, J. Gorjão Clara. Potentially inappropriate medicines use, by STOPP/START criteria, in a group of old patients admitted to a Portuguese hospital. European Geriatric Medicine 2013 sep, Volume 4, Supplement 1, S195

28. Ruscin MJ: Drug therapy in the elderly. In Porter RS \& Kaplan JL (eds): The Merck Manual, 3090-3098. Merck, Sharp \& Dohme Corp., 2011.

29. Ćurković M, Dodig-Ćurković K, Erić AP, Kralik K, Pivac N. Psychotropic medications in older adults: a review. Psychiatria Danubina, 2016; 28 (1): 13-24

30. Uzun S1, Kozumplik 0, Jakovljević M, Sedić B. Side effects of treatment with benzodiazepines. Psychiatr Danub. 2010 Mar; 22(1):90-3.

31. Dionne PA, Vasiliadis HM, Préville $M$. The economic impact attributable to the inappropriate prescription of benzodiazepines in the elderly living in the community. Value in Health, 2011-05-01; 14(3): A106-A106

32. Guthrie B, Clark SA, McCowan C. The burden of psychotropic drug prescribingin people with dementia: a populationdatabase study. Age and Ageing, 1 September 2010; 39(5): 637-642

33. Laheij RJF, Sturkenboom MCJM, Hassing RJ, et al: Risk of community-acquired pneumonia and use of gastric acid-suppression drugs. JAMA 2004; 292: 19551960

34. Leonard J, Marshall JK, and Moayyedi P: Systematic review of the risk of enteric infections in patients taking acid suppression. Am J Gastroenterol 2007; 102 2047-2056

35. Deshpande A, Pant C, Pasupuleti V, et al: Association between PPI therapy and Clostridium difficile Infection: A ContePPOrary Systematic Review and Meta-Analysis. Clin Gastroenterol Hepatol 2012; 10: 225-233

36. Sociedade Portuguesa de Reumatologia (SPR) e pela Sociedade Portuguesa de Doenças Ósseas Metabólicas. Recomendações para o diagnóstico e terapêutica da Osteoporose. 2007

37. Reid I.R., Bolland M.J., and Grey A.: Effects of vitamin D supplements of bone mineral density: a systematic review and meta-analysis. Lancet 2014; 343: pp. 146-155 